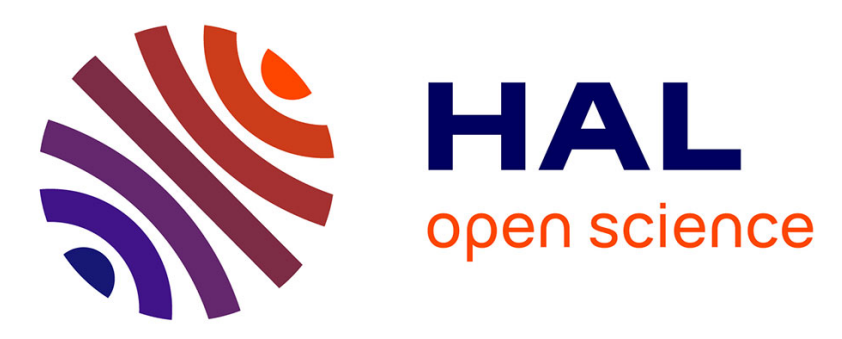

\title{
Robust Evaluation of Long-Term Stability of an InAs/GaSb Type II Superlattice Midwave Infrared Focal Plane Array
}

Vignesh Arounassalame, Maxence Guenin, Marcel Caes, Linda Hoglund, Eric Costard, Philippe Christol, Isabelle Ribet-Mohamed

\section{To cite this version:}

Vignesh Arounassalame, Maxence Guenin, Marcel Caes, Linda Hoglund, Eric Costard, et al.. Robust Evaluation of Long-Term Stability of an InAs/GaSb Type II Superlattice Midwave Infrared Focal Plane Array. IEEE Transactions on Instrumentation and Measurement, 2021, 70, pp.5001108. 10.1109/TIM.2020.3024406 . hal-03042939

\section{HAL Id: hal-03042939 https://hal.science/hal-03042939}

Submitted on 15 Mar 2021

HAL is a multi-disciplinary open access archive for the deposit and dissemination of scientific research documents, whether they are published or not. The documents may come from teaching and research institutions in France or abroad, or from public or private research centers.
L'archive ouverte pluridisciplinaire HAL, est destinée au dépôt et à la diffusion de documents scientifiques de niveau recherche, publiés ou non, émanant des établissements d'enseignement et de recherche français ou étrangers, des laboratoires publics ou privés. 


\title{
Robust evaluation of long term stability of an InAs/GaSb type II superlattice midwave infrared focal plane array
}

\author{
V. Arounassalame, M. Guénin, M. Caes, L. Höglund, E. Costard, P. Christol, I. Ribet-Mohamed
}

\begin{abstract}
The temporal stability of the image quality of an infrared focal plane array (FPA) is one key parameter to consider for high performance imaging applications. It is generally evaluated through Residual Fixed Pattern Noise (RFPN) measurements realized after a two-point correction (TPC) and bad pixels exclusion. However, the effect of random telegraph signal (RTS) on temporal stability has never been quantified, the intermittent blinking of RTS pixels making measurements inherently complicated. In this paper, we propose a novel protocol and a novel data processing technique to fully characterize the temporal stability of an infrared FPA, thanks to simultaneous RFPN and RTS noise measurements. Using a robust statistics based on Median Absolute Deviation (MAD) leads to a more trustful RFPN evaluation, unaffected by the bad pixels exclusion step. This new protocol can be applied to any FPA technology, cooled or uncooled, operating in the [3-14 $\mu \mathrm{m}]$ spectral domain. We chose to use a Type-II superlattice (T2SL) midwave infrared FPA integrated in a cryocooler at $80 \mathrm{~K}$. We show that the long-term stability of this $320 x 256$ pixels FPA is excellent. The RFPN only slightly increases over 77 days, using a TPC that has been calculated on the first day of the measurements campaign. A simple offset update, compatible with operational contingencies, can further improve the temporal stability of this FPA. Only $0.3 \%$ of the pixels were affected by RTS noise at some moment. Those blinking pixels don't affect the RFPN evolution, which is a very encouraging result for T2SL detector technology.
\end{abstract}

Index Terms - focal plane array, infrared detector, residual fixed pattern noise, performance evaluation, robust statistic, temporal stability, type-II superlattice.

TABLE 1. Key parameters used in the paper.

\begin{tabular}{|c|c|c|}
\hline Parameter & Acronym & Feature \\
\hline $\begin{array}{c}\text { Residual Fixed } \\
\text { Pattern Noise }\end{array}$ & RFPN & $\begin{array}{c}\text { Spatial image quality } \\
\text { after non uniformity } \\
\text { correction }\end{array}$ \\
\hline $\begin{array}{c}\text { Random } \\
\text { Telegraph Signal }\end{array}$ & RTS & $\begin{array}{c}\text { Low frequency noise that } \\
\text { creates jumps in the } \\
\text { signal }\end{array}$ \\
\hline $\begin{array}{c}\text { Median absolute } \\
\text { deviation }\end{array}$ & MAD & $\begin{array}{c}50 \% \text { confidence interval } \\
\text { robust calculation }\end{array}$ \\
\hline $\begin{array}{c}\text { RFPN calculated } \\
\text { with Median }\end{array}$ & RFPN $_{\text {MAD }}$ & $\begin{array}{c}\text { Standard deviation }(68 \% \\
\text { confidence interval) } \\
\text { robust calculation }\end{array}$ \\
\hline
\end{tabular}

Manuscript received May 28, 2020. This work was supported in part by the Agence Innovation Défense, from the French Ministry of Defence (MoD).

V. Arounassalame, M. Guénin, M. Caes and I. Ribet-Mohamed are with the ONERA, 91761 Palaiseau, France (e-mail : vignesh.arounassalame@onera.fr).

P. Christol is with Institut d'Electronique et des Systèmes (IES), Université de Montpellier, UMR CNRS 5214, 34095 Montpellier, France (e-mail: christol@ies.univ-montp2.fr).

\begin{tabular}{|c|c|c|}
\hline $\begin{array}{c}\text { Absolute } \\
\text { Deviation }\end{array}$ & & \\
\hline Two Point & TPC & Non uniformity \\
Correction & & $\begin{array}{c}\text { correction calculated with } \\
\text { two reference } \\
\text { temperatures }\end{array}$ \\
\hline
\end{tabular}

\section{INTRODUCTION}

$\mathrm{T}$ HERMAL infrared sensors are a wide field of study, thanks to a broad spectral range (from $3 \mu \mathrm{m}$ to $14 \mu \mathrm{m}$ ), multiple applications (military, aerospace, industrial ...), and different achievable technologies for the fabrication of one of the main parts of the sensor : the focal plane array (FPA). A growing demand for high performance sensors encouraged technological developments which aimed to propose detectors tailored for specific requirements given the intended application.

In the MWIR (3-5 $\mu \mathrm{m})$ and LWIR ( $8-14 \mu \mathrm{m})$ domains, several well-established high performance cooled or uncooled technologies are available. For example, quantum detectors can be based either on II-VI materials or on III-V materials [1]. Antimony-based type-II superlattice (T2SL) detectors are a recently matured III-V technology [2] which could combine the advantages of several technologies: the cut-off wavelength can be tailored thanks to band gap engineering, the electro-optical performance (especially quantum efficiency) is close to that of $\mathrm{HgCdTe}$ [3], high operating temperatures are accessible [4], and promising results in terms of spatial uniformity and operability have been reported [5]. This maturity enabled to study its potential for radiation thermometry for example [6].

The performance of infrared focal plane arrays is evaluated through commonly accepted figures of merit such as quantum efficiency or responsivity, completed by fixed pattern noise (FPN) and modulation transfer function (MTF). However, there is still a lack of appropriate and commonly accepted metrics to better assess the potential of a given technology for high performance imaging. For example, the temporal stability of the signal delivered by a focal plane array is of major importance.

L. Höglund and E. Costard are with Irnova AB, Electrum 236, SE-164 40 Kista, Sweden (e-mail : eric.costard@ir-nova.se). 
Indeed, it dictates how often the calibration of operational electro-optical systems has to be done, and thus reflects the availability of the system during an operational mission. In this way, long term stability could become a key merit function in technology comparison.

However, finding the appropriate figure of merit to quantify the temporal stability is not so easy. The commonly used residual fixed pattern noise (RFPN) is for example highly affected by the preliminary step consisting in excluding the bad pixels [7]. As a result, different criteria for bad pixels exclusion have been developed [8][9][10][11][12], making comparisons between detectors difficult. Moreover, this bad pixels exclusion step doesn't necessarily detect pixels with random telegraph signal (RTS) noise. The signal delivered by these pixels can oscillate between two (or more) levels at certain times, creating a particularly harmful blinking effect for image quality. To date, the possible link between RFPN degradation and the appearance of RTS pixels has not been reported.

This paper reports on the study of the temporal stability of a MWIR T2SL IDDCA (Infrared Detector with Dewar Cooler Assembly) from IRnova. A new experimental protocol was defined to realize simultaneously RTS and RFPN measurements, the latter being evaluated with a robust estimator. The new protocol and data processing enables to reach the true RFPN values even if there are some bad pixels or RTS pixels left in the pixels exploited. The method developed here can be applied for any infrared detector operating in the MWIR or LWIR spectral domains, cooled and uncooled.

\section{EXPERIMENTAL PROCEDURE}

The MWIR T2SL VGA/4 FPA with 320x256 pixels (30 $\mu \mathrm{m}$ pitch) used in this work is integrated in an IDDCA. TABLE 2 provides an overview of its specifications. The detector's signal output is coded on 16 bits, between $0.3 \mathrm{~V}$ and $2.7 \mathrm{~V}$.

The experimental bench consists of the T2SL IDDCA detector facing a CI-system SR80-4D/A blackbody (Fig. 1), hence the detector receives a controlled irradiance. The integration time of the FPA is set to $4 \mathrm{~ms}$ with a frame time of $16 \mathrm{~ms}(60 \mathrm{~Hz}$ frame rate). The blackbody's specifications are in TABLE 3.

The experiment is made to perform both RFPN measurements and RTS pixels detection, in order to see if the degradation of the RFPN is correlated with the apparition of blinking pixels. RFPN measurements require varying the blackbody's temperature (to explore different well fill values of the integrating capacitor), while the study of RTS pixels requires long acquisitions at fixed blackbody temperature instead. The following protocol has therefore been established (see Fig. 2) : the blackbody temperature is varied from $10^{\circ} \mathrm{C}$ to $58^{\circ} \mathrm{C}$ with a $3^{\circ} \mathrm{C}$ step ( 17 cubes of images). These temperatures were chosen as they were easily accessible with an extended blackbody, integration time was then adjusted accordingly at 4 $\mathrm{ms}$ in order to reach the saturation for the highest blackbody's temperature.

For each temperature, a cube of 5000 images is acquired. The first 256 images are used to infer the RFPN (temporally averaged image is used for the calculation), whereas the whole cube is analysed to extract RTS pixels. The number of 5000 images is chosen as a good compromise between observation time and data processing complexity. In order to study the time evolution of the RFPN and RTS pixels, this measurement was repeated day after day, sometimes within the same cooling cycle and sometimes after letting the FPA warm up to room temperature and a new cooling cycle.

Data from two measurements campaigns have been used. Indeed, a pause of 45 days passed exactly between the two campaigns of measurements, in order to have a long time evaluation. A total of 74 RFPN measurements (one RFPN measurement equals to 17 cubes of images) were realized, representing a cumulated acquisition time of 28 hours. Between the first and last RFPN measurements, 77 days have passed.

TABLE 2. Specifications of the IRnova FPA under test.

\begin{tabular}{|c|c|}
\hline Format & $320 \times 256$ \\
\hline Pixel pitch & $30 \mu \mathrm{m}$ \\
\hline Spectral Range & $3.5-5 \mu \mathrm{m}$ \\
\hline F number & $\mathrm{F} / 2$ \\
\hline $\begin{array}{c}\text { Noise Equivalent Temperature Difference } \\
\text { (NETD) }\end{array}$ & $12 \mathrm{mK}$ \\
\hline Operability & $99.8 \%$ \\
\hline Operating Temperature & $80 \mathrm{~K}$ \\
\hline Framerate & $60 \mathrm{~Hz}$ \\
\hline
\end{tabular}

TABLE 3. Specifications of the blackbody.

\begin{tabular}{|c|c|}
\hline Temperature range & {$\left[5^{\circ} \mathrm{C}-100^{\circ} \mathrm{C}\right]$} \\
\hline Uniformity & $\pm 0,01^{\circ} \mathrm{C}$ \\
\hline Stability & $\pm 0,02^{\circ} \mathrm{C}$ \\
\hline Emissivity & $0.97 \pm 0,02$ \\
\hline Dimensions (WxLxH) & $161 \mathrm{~mm} \times 155 \mathrm{~mm} \times 165 \mathrm{~mm}$ \\
\hline Emitting surface & $106 \mathrm{~mm} \times 106 \mathrm{~mm}$ \\
\hline
\end{tabular}

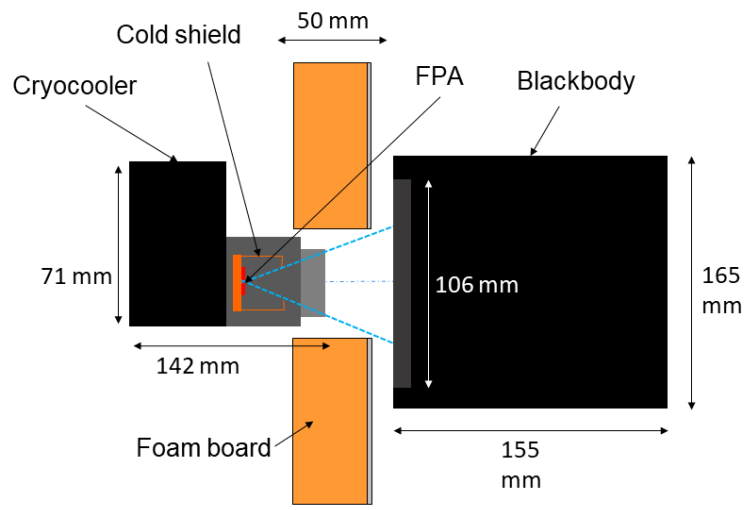

Fig. 1. Representation of the experimental bench. On the right the blackbody whose temperature varies between $10^{\circ} \mathrm{C}$ and $58^{\circ} \mathrm{C}$. On the left, the $\mathrm{T} 2 \mathrm{SL}$ IDDCA. A cold shield defines the field of view (FOV) of the FPA, and the cryocooler cools down the FPA to $80 \mathrm{~K}$. A piece of pierced foam is placed between the blackbody and the detector to reduce thermal convection.

\section{DATA PROCESSING}

The Fixed Pattern Noise corresponds to the spatial fluctuations of the signal delivered by the pixels composing an FPA when it receives a uniform irradiance. The fixed pattern noise can find its origin in two different parts of the FPA: the first one is the detection circuit made of T2SL technology 
(which converts photons into electrons) because of differences in cut-off wavelengths for example. The second one is the Read Out Integrated Circuit made of silicon (ROIC, which processes the pixel data to generate a video signal) for example if the output amplifiers have different offsets.

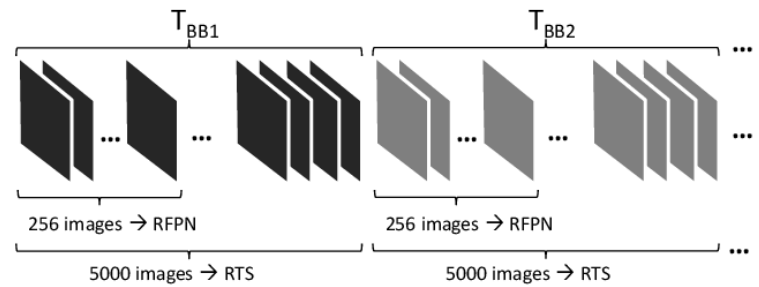

Fig. 2. Experimental protocol. Cubes of 5000 images are acquired for 17 values of the blackbody temperature. The first 256 images of each cube are used for RFPN measurements, while the 5000 images are used for RTS noise study.

\section{A. Two-point correction}

A linear two-point correction (TPC) [13] is commonly realized on the signal in order to reduce the Fixed Pattern Noise. This calibration is done using two extended blackbodies at different temperatures $T_{1}$ and $T_{2}$. The corrected signal for one pixel $(\mathrm{i}, \mathrm{j}) S_{i, j}^{\prime}$ is calculated using the two coefficients of the linear correction $G_{i, j}$ and $O_{i, j}$ :

$$
\left\{\begin{array}{c}
S_{i, j}^{\prime}(\phi)=G_{i, j} S_{i, j}(\phi)+O_{i, j} \\
S_{i, j}^{\prime}\left(\phi_{1}\right)=\left\langle S\left(\phi_{1}\right)\right\rangle \\
S_{i, j}^{\prime}\left(\phi_{2}\right)=\left\langle S\left(\phi_{2}\right)\right\rangle
\end{array}\right.
$$

where $S_{i, j}(\phi)$ is the signal delivered by the pixel $(\mathrm{i}, \mathrm{j})$ for an incident power equal to $\phi$ (after removing the electrical offset of the pixel). $\phi_{1}$ and $\phi_{2}$ correspond to the incident powers of the two blackbodies. They are chosen such as the Well Fill reaches $33 \%$ and $66 \%$ respectively. They enable to have an efficient correction on the whole detector's dynamic. Other values can be chosen depending on the application. $\left\langle S\left(\phi_{1}\right)\right\rangle$ (respectively $\left\langle S\left(\phi_{2}\right)\right\rangle$ ) represents the spatially averaged signal at the incident power $\phi_{1}$ (respectively $\phi_{2}$ ). The two correction coefficients, gain and offset respectively, are calculated from (2) and (3) assuming that each pixel has a linear signal with the incident power:

$$
\begin{gathered}
G_{i, j}=\frac{\left\langle S\left(\phi_{2}\right)\right\rangle-\left\langle S\left(\phi_{1}\right)\right\rangle}{S_{i, j}\left(\phi_{2}\right)-S_{i, j}\left(\phi_{1}\right)} \\
O_{i, j}=\left\langle S\left(\phi_{1}\right)\right\rangle-G_{i, j} S_{i, j}\left(\phi_{1}\right)
\end{gathered}
$$

This type of calibration is done only once, the correction coefficients are used for the RFPN measurements made afterwards to determine how long they remain valid.

\section{B. Offset Update correction}

Another type of correction is the offset update correction [14]. It is used in complementary of the TPC. Indeed, the TPC calculates $G_{i, j}$ and $O_{i, j}$ at the beginning, then before each RFPN measurement, the $O_{i, j}$ coefficient is refreshed. The following calculation is used :

$$
O_{i, j}=\left\langle S\left(\phi_{1}\right)\right\rangle-G_{i, j}^{t_{0}} S_{i, j}\left(\phi_{1}\right)
$$

with $G_{i, j}^{t_{0}}$ the Gain from the first RFPN measurement with the TPC. Hence, only $O_{i, j}$ is updated and the Gain used for the TPC is from the first RFPN measurement. Reminder, $\phi_{1}$ corresponds to an incident power such as the Well Fill (WF) capacity of the detector reaches $33 \%$. This type of complementary correction is often operationally accessible. Indeed, it can be performed if the operator uses the camera's shutter as a blackbody for the calibration before utilization. Therefore, we have chosen ambient temperature which corresponds to a WF of $33 \%$ in our case for the offset update.

\section{Temporal stability definition}

The two-point correction is very effective at the time it is implemented. However, the problem is to know if the correction used is appropriate for a long time. That is why, the Residual Fixed Pattern Noise (RFPN) which remains after the TPC is calculated. It indicates the effectiveness of the reduction of the Fixed Pattern Noise by its definition itself:

$$
\operatorname{RFPN}(\phi)=\sqrt{\frac{1}{N} \sum_{i} \sum_{j}\left(S^{\prime}{ }_{i, j}(\phi)-\left\langle S^{\prime}(\phi)\right\rangle\right)^{2}}
$$

where $\mathrm{N}$ is the total number of pixels of the FPA.

The RFPN is thus a standard deviation representing the global behavior of the FPA and it is usually interpreted as the thickness of the Gaussian distribution expected for photon noise limited pixels.

The RFPN tends to increase with time. This may be due to instabilities or drifts in the detector as well as in the instrument in which it is integrated. Determining when the TPC needs to be calculated again is not easy because it depends on the application. One can use the ratio of the RFPN over the temporal noise (TN) [15], the latter being defined as the temporal fluctuations of the signal delivered by a pixel. Indeed, fixed pattern noise and temporal noise are in general quadratically summed [16]. Therefore, the criterion that the RFPN must be inferior to the temporal noise in order to get a good image quality is reasonable. This ratio defines the temporal stability of the FPA.

\section{Bad pixels detection}

Before calculating the RFPN, some pixels have to be excluded because their behavior deviates too far from the whole FPA. Generally speaking, pixels whose mean level, response, noise and/or NETD (noise equivalent temperature difference) deviate too far from the median value are discarded (see TABLE 4), but the exact criteria vary from one team to another, which can have a strong influence on the RFPN value [7].

In this article, bad pixels are pixels which do not follow the main statistical distribution of the matrix. Among them can be found, hard defects pixels (dead pixels, short circuited pixels) and pixels which do not respond to the criteria used in TABLE 4. 
TABLE 4- Criteria used to classify one pixel as a bad one, $S_{i, j}^{\prime}$ is the corrected signal from the pixel, $\left\langle\mathrm{S}^{\prime}\right\rangle$ is the spatially averaged corrected signal, $\sigma_{S^{\prime}}$ is the spatial standard deviation of the corrected signal, $\sigma_{t i, j}$ is the temporal noise of the pixel, $\left\langle\sigma_{t}\right\rangle$ is the spatially averaged value, $\mathrm{R}_{i, \mathrm{j}}^{\prime}$ is the responsivity of the pixel and $\left\langle\mathrm{R}^{\prime}\right\rangle$ is the spatially averaged value, $\operatorname{NETD}_{i, j}^{\prime}$ is the NETD of the pixel after correction and $\left\langle\mathrm{NETD}^{\prime}\right\rangle$ is the spatially averaged value

\begin{tabular}{|c|c|c}
\hline & Criteria & $\begin{array}{c}\text { Number of } \\
\text { pixels }\end{array}$ \\
\hline Continuous level & $\left|S_{i, j}^{\prime}-\left\langle S^{\prime}\right\rangle\right|>10 .\left\langle\sigma_{S^{\prime}}\right\rangle$ & 43 \\
\hline Temporal Noise & $\left|\sigma_{t i, j}-\left\langle\sigma_{t}\right\rangle\right|>30 \% .\left\langle\sigma_{t}\right\rangle$ & 25 \\
\hline Responsivity & $\left|R_{i, j}^{\prime}-\left\langle R^{\prime}\right\rangle\right|>30 \% .\left\langle R^{\prime}\right\rangle$ & 34 \\
\hline NETD & $\begin{array}{c}\left|N E T D^{\prime}{ }_{i, j}-\left\langle N E T D^{\prime}\right\rangle\right| \\
>100 \% .\left\langle N E T D^{\prime}\right\rangle\end{array}$ & 37 \\
\hline Total & & 49 \\
\hline
\end{tabular}

256 images were acquired on day 1 as specified by the protocol detailed in the previous section. The total number of bad pixels detected is 49 , which corresponds to an operability of $99.94 \%$. This total is not equal to the sum of the number of pixels in each category, because one bad pixel can have multiple defects and fall simultaneously into several categories.

Once the bad pixels have been set aside, the residual fixed pattern noise is calculated on the corrected images (after application of the two-point correction) thanks to equation (5).

\section{E. RFPN evaluated with Median Absolute Deviation (MAD)}

Even if the selection of bad pixels is careful, some pixels with atypical behavior may persist and have a significant influence on the RFPN. This is due to the fact that the RFPN evaluation relies on a standard deviation calculation, which is well known to encounter problems regarding outliers.

To tackle this issue, the median absolute deviation (MAD) [17] can be used as a robust estimator. Indeed, using the median value rather than the mean value as in equation (6) provides a robust measure of statistical dispersion. The MAD is very insensitive to the presence of outliers. One indicator of this insensitivity is the "breakdown point" [18]. The estimator's breakdown point is the maximum proportion of data points that can be outliers without forcing the estimator to result in a false value. For the MAD, this value is 50\%. It means that as long as less than half of the data points are outliers, the MAD will have the correct value. The MAD is defined as follows:

$$
M A D=\operatorname{median}\left(\left|S^{\prime}-\operatorname{median}\left(S^{\prime}\right)\right|\right)
$$

With S' the corrected image. But MAD is only a robust measure of the variability of sample data, and does not represent the standard deviation. A scale factor $k$ [19] must be applied in order to define the corresponding RFPN :

$$
\operatorname{RFPN}_{M A D}=k \cdot M A D
$$

Where $\mathrm{k}$ is a constant scale factor, which depends on the distribution. Usually, $\mathrm{k}$ is taken equal to 1.4826 , under the assumption of normality of the data, without taking into account the outliers.
The importance of a robust estimator can be observed on Fig. 3. Indeed, the robust RFPN $\mathrm{NAD}_{\mathrm{M}}$ is clearly lower than the RFPN polluted by outliers. It can be noted that both the RFPN $\mathrm{N}_{\text {MAD }}$ and the RFPN polluted by outliers exhibit the usual "W" shape curve, making the effect of outliers difficult to detect.

\section{F. Random Telegraph Signal (RTS) pixels}

Recent attempts to increase the operating temperature of infrared focal plane arrays and to reduce their size, weight and power (SWaP) have shown that image quality could be degraded due to pixels affected by RTS (random telegraph signal) noise [20][21][22].

As can be seen in Fig. 4, those RTS pixels exhibit a signal oscillating between at least two levels, while the received power

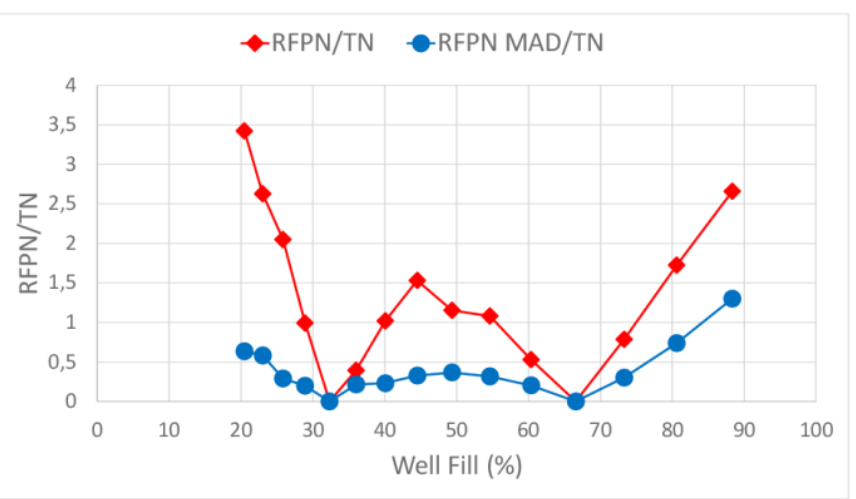

Fig. 3. RFPN/TN and RFPN MAD/TN over the Well Fill calculated on a data set containing two outliers.

and operating conditions are unchanged. This results in a blinking effect as a function of time, which cannot be suppressed by TPC. Since the blinking is not permanent, detecting these pixels is a very difficult task and is the subject of intensive research [23]-[24]. The algorithm used here for the detection of RTS pixels is from [25]. The main steps of the algorithm are:

- A list of candidate pixels is calculated using a criterion on temporal noise.

- For each candidate pixel, the temporal evolution of the signal (also called continuous level) is analyzed by the Pruned Exact Linear Time (PELT) algorithm [26] in order to detect the jumps and level differences. The principle relies on changepoints detection of the continuous level's mean value. These changepoints are detected by using a cost function from the PELT algorithm.

- Finally, a candidate pixel is confirmed as a RTS pixel if the difference of levels of the jumps is superior to the temporal noise.

\section{RTS PIXELS ANALYSIS}

RTS pixels are detected using the algorithm from [25] and then classified. After exclusion of hard defects and continuous level defects, 280 pixels are identified as RTS pixels. However, RTS pixels' origins are not known in this FPA. Indeed, in an infrared FPA, RTS pixels can have different origins: either from the detection circuit which is composed of T2SL material or the ROIC which is in silicon. 


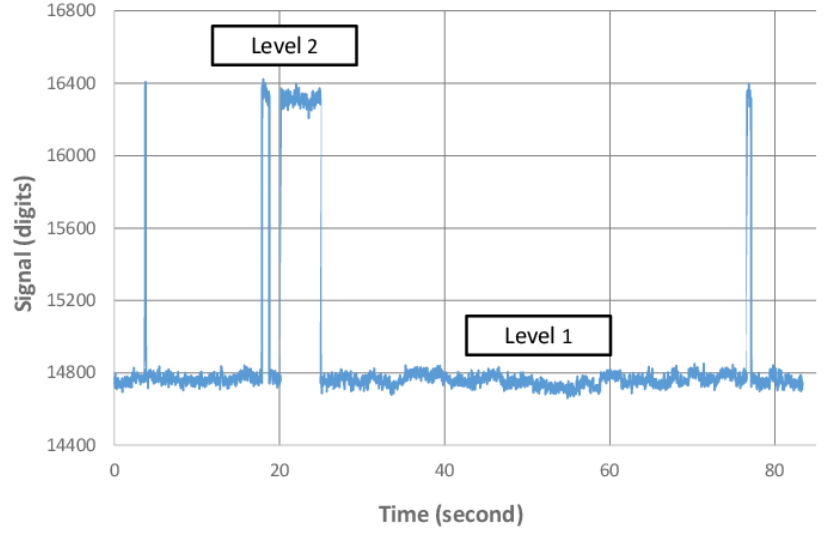

Fig. 4. Raw signal (in digits) as a function of time, for a RTS pixel facing a blackbody whose temperature is stable over time. This RTS pixel is categorized as a 2-level RTS pixel. Level 1 refers to the low level and level 2 to the high level.

An experiment is made to distinguish if RTS pixels come from the ROIC or the detection circuit. In order to isolate RTS pixels coming only from the ROIC, measurements (cubes of 5000 images) are made at a near zero integration time $(1 \mu \mathrm{s})$. A first list of RTS pixels is computed, and then the definitive list is made from the common RTS pixels between the first list and the list of RTS pixels detected at $4 \mathrm{~ms}$. This comparison is made in order to know which RTS ROIC pixels were still observable under flux. The results show that only 22 RTS pixels under flux come from the ROIC, and 21 among them belong to hard defects or continuous levels defects.

RTS ROIC pixels are now excluded, in order to focus the study on T2SL RTS pixels. 279 RTS pixels remain. These pixels are divided into three categories :

- Two-levels: RTS pixel with a continuous signal oscillating between two levels at least on one measurement ( 251 out of 279 RTS pixels, hence $90 \%$ ).

- Multi-levels: RTS pixel with a continuous signal oscillating between multiple levels (more than two) at least on one measurement $(0.4 \%)$.

- Noisy: RTS pixel whose continuous signal has too much noise to clearly identify different levels $(10 \%)$.

In the following, only two level RTS pixels are considered for the sake of simplicity. Long and numerous measurements (see II-Experimental Procedure) are realized in order to see if a fixed population of RTS pixels exists. If so, then excluding those RTS pixels could help to solve the problem of image quality deterioration. Indeed, if these pixels are identified, their signals can be replaced by their immediate neighbors thanks to image processing. That is the purpose of the 74 measurements, with each measurement containing $664 \mathrm{~s}$ (8x83s) of cumulated observation time data. Only the 8 first blackbody's temperatures (from $10^{\circ} \mathrm{C}$ to $31^{\circ} \mathrm{C}$ ) are exploited, because high blackbody's temperatures induce important shot noise which hides some RTS noises. Fig.5 shows two curves: one is the number of RTS pixels detected for each measurement, the other is the cumulated number of RTS pixels, i.e. the number of pixels which blinked at least once since the beginning of measurements. The black rectangle between measurements 24 and 25 represents the 45 days of pause between both measurement campaigns.

The number of pixels which were 2-level RTS pixels at some point is 251 (i.e $0.3 \%$ of the FPA), in 13.7 hours of cumulated observation time. In average, there were 10 RTS pixels by measurement, which is very low and steady. The number of cumulated RTS pixels does not seem to reach a plateau, meaning that there is no fixed population of RTS pixels. Because of the numerous thermal cycles, it is difficult to see if there is a difference on the number of new RTS pixels appearing after each measurement. That is why, measurements are divided into two categories: measurements realized within a given cooldown and measurements realized after letting the FPA reach room temperature (new cooling cycle). Statistics in TABLE 5 show that the appearance of RTS pixels is correlated to thermal cycles. Indeed, there are five times more new RTS pixels appearing after letting the FPA reach room temperature than during the same cooldown.

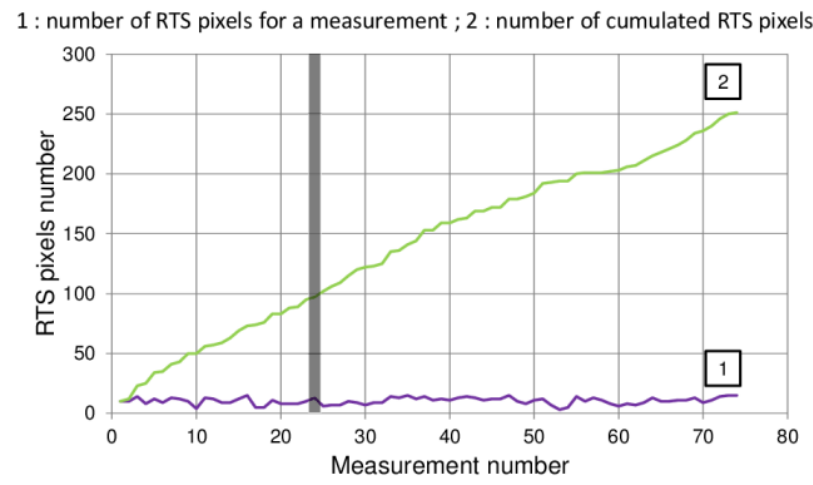

Fig.5. 2-levels RTS pixels detected for each measurement of RFPN. The purple curve is the absolute number of RTS pixels detected at each measurement. The green curve represents the cumulated number of RTS pixels. The black rectangle represents the 45 days of pause between both campaigns.

TABLE 5 - Statistics of the number of new 2-level RTS pixels appearing, within a given cooldown, after letting the FPA reach room temperature, and between two consecutive measurements (regardless of thermal cycles).

\begin{tabular}{|c|c|c|}
\hline $\begin{array}{c}\text { Number of new RTS pixels } \\
\text { between two consecutive } \\
\text { measurements: }\end{array}$ & Average & $\begin{array}{c}\text { Standard } \\
\text { deviation }\end{array}$ \\
\hline Within a thermal cycle & 1 & 2 \\
\hline After a new thermal cycle & 5 & 3 \\
\hline Regardless of thermal cycles & 3 & 3 \\
\hline
\end{tabular}

\section{RFPN ANALYSIS}

The evolution of the RFPN/TN ratio over time (or number of RFPN measurements here) is studied in order to evaluate the temporal stability of the FPA.

Fig.6. represents the RFPN $\mathrm{MAD}_{\mathrm{MAN}} / \mathrm{TN}$ over the Well Fill at different times using the TPC from day 1 . As it can be observed, $\mathrm{RFPN}_{\mathrm{MAD}} / \mathrm{TN}$ corresponding to day 1 shows the best performance without surprise, since the TPC corresponds to the data set at that time. As expected, the $\mathrm{RFPN}_{\mathrm{MAD}} / \mathrm{TN}$ cancels itself out at the reference points which correspond to $33 \%$ and 
$66 \%$ of the Well Fill. But as times passes, the TPC loses its effectiveness as it can be seen on the curves representing days 13 and 77. Fig.7. represents the $\mathrm{RFPN}_{\mathrm{MAD}} / / \mathrm{TN}$ with the offset update. The improvement is important. Until $50 \%$ of the Well Fill, there is practically no difference with day 1 . After $50 \%$ of the Well Fill, there is an increase of the $\mathrm{RFPN}_{\mathrm{MAD}} / \mathrm{TN}$, however, the increase is less important than without offset update. It can be interpreted that the main factor to the pixels' signal variation in time is only a shift, that is why the offset updates are very efficient.

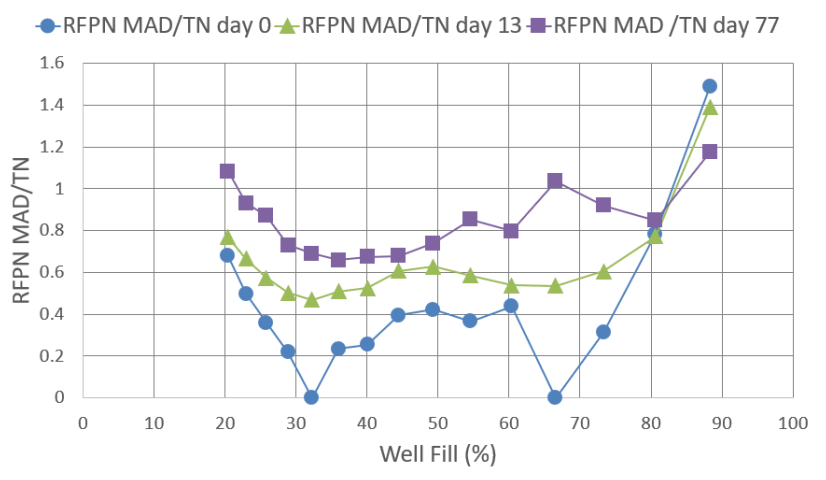

Fig.6. RFPN MAD/TN over the Well Fill at different times, using the TPC from the measurement day 1 .

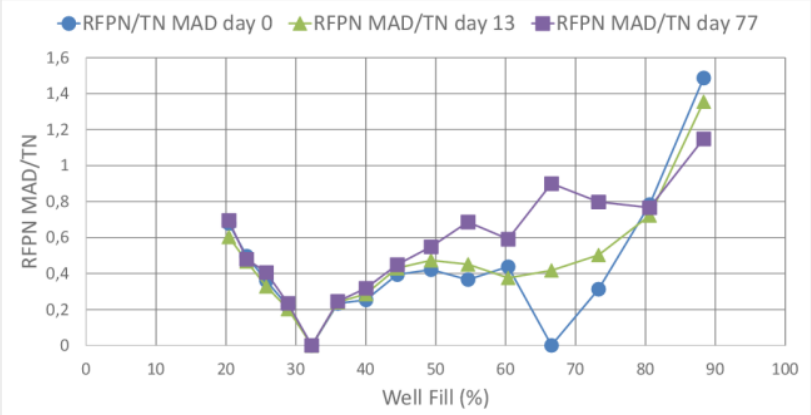

Fig.7. RFPN MAD/TN over the Well Fill at different times, using the TPC from the measurement day 1 and complementary offset update.

The previous analyses were made for three measurements, for the sake of clarity. The whole data set is analyzed in the following discussions through the time evolution of the

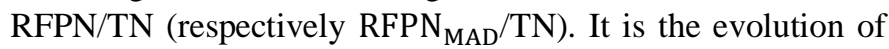
the RFPN/TN (respectively RFPN $\mathrm{MAD}_{\mathrm{M}} / \mathrm{TN}$ ) evaluated at $50 \%$ of the Well Fill. The black rectangle between measurements 24 and 25 represents the 45 days of pause between both measurements.

In Fig.8 the time evolution of the RFPN/TN is represented. It is focused on determining the potential role of RTS pixels in degrading the temporal stability. For each evolution, the calibration is done on measurement one: the TPC correction coefficients are calculated and bad pixels are detected and excluded. Then, for each measurement after calibration, the correction coefficients calculated are applied. The diamond dots curve is the evolution of the ratio RFPN/TN without detection of RTS pixels for any measurement. As for the square dots curve, the same is applied but for each measurement, RTS pixels are detected and excluded from data exploitation. Before comparing the two curves, it can be noted firstly, that for the whole duration of the measurements, the RFPN/TN remains lower than one. Secondly, there is a sudden important increase of $60 \%$ after measurement 2 , but the ratio quickly becomes relatively stable. Moreover, the RFPN/TN is not constantly increasing, it is also dropping sometimes from one measurement to another. Contrarily to what can be thought, the image quality is not growingly deteriorated. Sometimes it is improved compared to the previous measurement as it can be seen between measurements 20 and 21 . These "relaxation phenomenon" of the RFPN/TN happen when the detector has been switched off for 15 hours.

At last, the comparison between the two curves shows that there is almost no difference between them. There are no sudden increases due to RTS pixels and there is not a constant shift throughout measurements between the two quantities Hence, RTS pixels for a detector temperature of $80 \mathrm{~K}$ are not responsible for the deterioration of global image quality. Here it only has a slight effect on the RFPN/TN because there are only a few ones, their amplitude is weak and their frequency of apparition is low. However, it is known that increasing the temperature's detector increases the number of RTS pixels, and a deterioration of the global image quality can happen because of RTS pixels. That is why, as underlined in section III, the MAD criterion has to be utilized in order to ensure that the true image quality is evaluated. In order to properly evaluate RTS pixels impact when there are only a few ones, a way to evaluate the local image quality will have to be found.

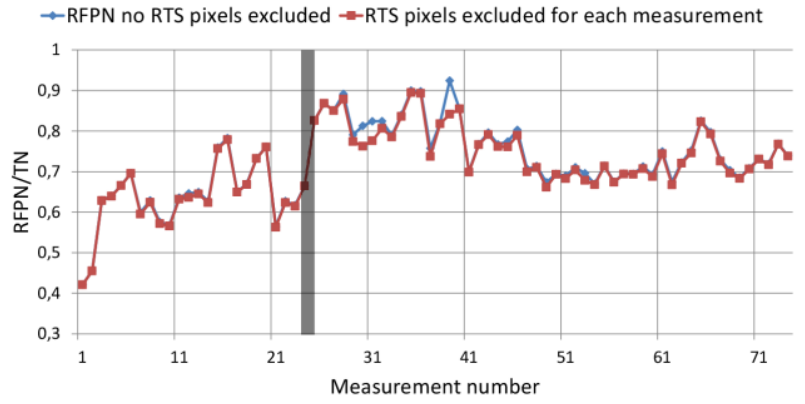

Fig.8. Comparison of the time evolution (in number of measurements) of RFPN with and without RTS pixels excluded. The black rectangle represents the 45 days of pause between both campaigns, it explains the discontinuity observed.

In Fig.9, RFPN MAD $/ \mathrm{TN}$ and RFPN/TN are compared. The $\mathrm{RFPN}_{\mathrm{MAD}} / \mathrm{TN}$ is inferior to the RFPN/TN, as expected, thanks to its robustness. However, the two quantities are very close and excluding more pixels to reach the $\mathrm{RFPN}_{\mathrm{MAD}} / \mathrm{TN}$ value could be counterproductive, since good pixels could be eliminated as well, for a small improvement. The comparison between $\mathrm{RFPN}_{\text {MAD }}$ and RFPN improvement in favor for the last one. The offset update enables to have an almost constant RFPN throughout the whole measurement. However, it can be noted that the measurements 39 to 47 and 60 to 65 have a sudden increase compared to other measurements and it does not reappear in later measurements. In order to understand what phenomenon caused this increase, the temporal noise of the FPA against the blackbody's 
temperature was studied. And it was seen that the temporal noise for temperatures between $31{ }^{\circ} \mathrm{C}$ and $55^{\circ} \mathrm{C}$ is very important when the problem occurred, compared to other measurements. Indeed, it does not follow the evolution of the shot noise as it should be expected. Moreover, the observation of the temporal noise image shows that it is inhomogeneous. In view of the time constants characteristic of the phenomenon we concluded that convection occurred between the detector and the blackbody for these measurements. That is why this part should not be considered for the interpretation of temporal stability.

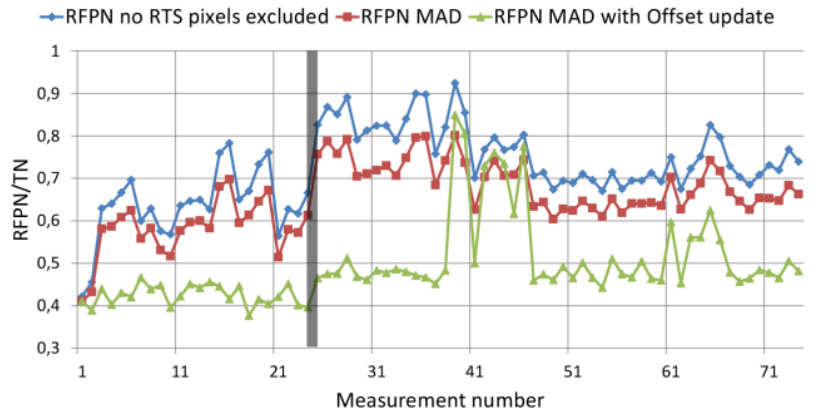

Fig.9. Comparison of the time evolution (in number of measurements) of RFPN, RFPN MAD and RFPN MAD with offset update. The black rectangle represents the 45 days of pause between both campaigns.

Finally, there is one last important accessible parameter that was left untouched: the number of images used for averaging. Indeed, this parameter is important because it determines if the detector can be used in operational condition or not. Moreover, the FPN is a very sensitive quantity, which can fluctuate because of the environment (room temperature, parasitic flux, hygrometry...). That is why it is hard to give a clear interpretation of fluctuations of the RFPN/TN ratio under one. Hence, the time evolution of the $\mathrm{RFPN}_{\mathrm{MAD}} / \mathrm{TN}$ for 1 image instead of 256 is observed in Fig.10.

The $\mathrm{RFPN}_{\mathrm{MAD}} / \mathrm{TN}$ becomes superior to one, but it stays between values 1.1 and 1.3 for the whole time. As for the offset update method, the improvement is modest ( $8 \%$ of decrease compared to the $\mathrm{RFPN}_{\mathrm{MAD}} / \mathrm{TN}$ without offset update). However, it is very stable and there is almost no variation, exception made of the measurements when the convection occurred. These results are encouraging for the temporal stability and uniformity of this detector.

\section{CONCLUSION}

We have presented a new experimental protocol and the associated data processing technique to fully characterize the temporal stability of the image quality of infrared focal plane arrays.

The experimental protocol has been defined so as to carry out simultaneous RFPN and RTS noise measurements. We thus combine measurements varying the blackbody temperature (to extract RFPN) and long acquisitions at fixed blackbody temperature (to study RTS pixels). Measurements are repeated, either within one cooling cycle or

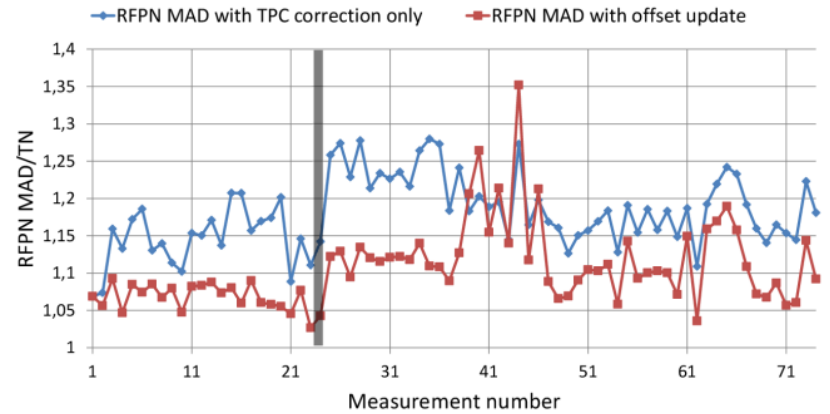

Fig.10. Time evolution RFPN MAD/TN calculated on 1 image instead of an averaging of 256 images. The black rectangle represents the 45 days of pause between both campaigns.

after letting the detector reach room temperature and a start new cooling cycle, in order to rule on a possible correlation between cooling cycles and RFPN / RTS pixels evolution. In order to overcome the limitations of the usual RFPN figure of merit, which is strongly affected by outliers and thus intrinsically depends on the bad pixels exclusion step, we have defined a robust estimator based on the median absolute deviation.

Using this new capacity, we have realized the first simultaneous RFPN and RTS noise measurements on an infrared focal plane array. The device under test was a commercial T2SL MWIR $320 \times 256$ pixels focal plane array integrated in a cooler assembly operating at $80 \mathrm{~K}$. Our results show that the temporal stability is excellent, with a remarkably stable RFPN up to 77 days, applying a TPC that was calculated on the first day. The number of RTS is very low, with 10 blinking pixels in average by measurement. From one measurement to the next, the RTS pixels are not necessarily the same, such that the number of pixels that have blinked at least once since the beginning of the measurement keeps increasing, leading to a total of 251 pixels ( $0.3 \%$ of the $320 \times 256$ pixels) in 13.7 hours of cumulated observation time. Our results allow us to conclude that RTS pixels do not affect the RFPN evolution of this FPA, which, to the best of our knowledge, has never been demonstrated before. To finish with, we show that a simple offset update correction, which is generally available in operation, leads to an even better temporal stability. This focal plane array is also suitable for real time imaging, since RFPN remains close to one when it is evaluated on a single image.

We believe the exciting new measurement capacity demonstrated here will allow detector manufacturers to better understand the physical origins of temporal instabilities in infrared focal plane arrays. It will also help system integrators in setting detector specifications for camera cores and delivery compliance testing. To finish with, it opens the path to a rigorous comparison of the temporal stability of image quality of infrared focal plane arrays from different technologies.

\section{REFERENCES}

[1] A. Rogalski, "Infrared systems fundamentals", in Infrared and Terahertz Detectors, 3rd ed., vol. 3, New York, NY, USA: CRC Press Book, 2019, pp.21-47.

[2] A. Rogalski, P. Martyniuk, M. Kopytko, "InAs/GaSb type-II superlattice infrared detectors: three decades of development", in Infrared Technology and Applications XLIII, Anaheim, CA, USA, 2017.

[3] D. Wu, Q. Durlin, A. Dehzangi, Y. Zhang, M. Razeghi, "High quantum efficiency mid-wavelength infrared type-II InAs/InAs1- xSbx superlattice photodiodes grown by metal-organic chemical vapor 
deposition", Appl. Phys. Lett., vol. 114, no. 1, Jan. 2019. DOI : 10.1063/1.5058714

[4] D. Z. Ting, et al., "Mid-wavelength high operating temperature barrier infrared detector and focal plane array", Appl. Phys. Lett., vol. 113, no. 2, May 2018, DOI: 10.1063/1.5033338.

[5] L. Höglund, et al, "Manufacturability of type-II InAs/GaSb superlattice detectors for infrared imaging", Infrared Phys. \& Techn. vol. 84, pp. 2832, Aug. 2017. DOI: 10.106/j.infrared.2017.03.002.

[6] M. J. Hobbs, et al., "InAs/GaSb Type-II Superlattice for Radiation Thermometry", IEEE Trans. Instrum. Meas., vol. 64, no. 2, pp. 502-508, Feb. 2015. DOI: 10.1109/TIM.2014.2341411.

[7] I. Ribet-Mohamed et al, "Temporal Stability and correctability of a MWIR T2SL focal plane array ", Infrared Phys. \& Techn., vol.96, pp.145-150, Jan. 2019. DOI: 10.106/j.infrared.2018.10.028.

[8] R. Breiter., et al., "State-of-the-art MCT IR-modules with enhanced long term and cycle stability." in Infrared Technology \& Application XXXVIII., Baltimore, MD, USA, May 2012. DOI: 10.1117/12.919243.

[9] J. A. Robo, et al., "QWIP focal plane arrays performances from MWIR up to VLWIR. "in Infrared Technology and Applications XXXV, Orlando, FLA, USA, May 2009. DOI: 10.1117/12.820470.

[10] P. Delaunay, et al., "Advances in III-V based dual-band MWIR/LWIR FPAs at HRL.", in Infrared Technology and Applications XLIII, vol. 10177, Anaheim, CA, USA, May 2017. DOI: 10.1117/12.2266278.

[11] L. Rubaldo, et al., "State of the art HOT performances for Sofradir II-VI extrinsic technologies.", in Infrared Technology and Applications XLII, vol. 9819, Baltimore, MD, USA, May 2016. DOI: 10.1117/12.2229308

[12] H. Martijn et al., "QWIPs at IRnova, a status update.", Infrared Technology and Applications XLII, vol. 9819, Baltimore, MD, USA, May 2016. DOI: $10.1117 / 12.2228348$

[13] M. Schulz and L. Caldwell, "Non uniformity correction and correctability of infrared focal plane arrays", Infrared Phys. \& Techn., vol.36, no. 4, pp. 763-777, Aug. 1995. DOI: 10.106/1350-4495(94)00002-3.

[14] T. Orzanowski, "Nonuniformity correction algorithm with efficient pixel offset estimation for infrared focal plane arrays," SpringerPlus, vol. 5, Oct. 2016. DOI: 10.1186/s40064-016-3534-1.

[15] D.L. Perry and E.L. Dereniak, "Linear theory of nonuniformity correction in infrared staring sensors," Opt. Eng., vol. 32, no. 8, Aug. 1993. DOI: $10.1117 / 12.145601$.

[16] J.M. Mooney, F.F. Shepherd, W.S. Ewing, J.E. Ewing, J. Silverman, "Responsivity non-uniformity limited performance of infrared staring cameras", Opt. Eng., vol. 28, no. 11, Nov. 1989. DOI: $10.1117 / 12.7977112$

[17] P.J. Huber, "Scale estimates" in Robust statistics, vol.523, Hoboken, NJ, USA: JWS, 2004, pp.107-122.

[18] D.L. Donoho, P.J. Huber, "The notion of breakdown point", A festschrift for Erich L. Lehmann, vol.157184, 1983

[19] P.J. Rousseeuw and C. Croux, "Alternative to the median absolute deviation", J. Am. Stat. Assoc., vol. 88, no. 424, pp. 1273-1283, Feb. 1993.

[20] A.I. D'Souza, et al. "Noise attributes of LWIR HDVIP HgCdTe detectors ", J. Electron. Mater., vol. 37, pp.1318-1323, April 2008. DOI: 10.1007/s11664-008-0432-5.

[21] A. Brunner, et al. "Improvement of RTS noise in $\mathrm{HgCdTe}$ MWIR detectors", J. Electron. Mater., vol. 43, pp.3060-3064, June 2014. DOI: 10.1007/s11664-014-3214-z.

[22] D. Pogány and G. Guillot, "Random Telegraph Signal noise instabilities in lattice-mismatched InGaAs/InP photodiodes", Microelectron. Reliab., vol. 39, no. 3, pp.341-345, March 1999. DOI: 10.1016/S00262714(99)00007-4.

[23] Y. Yuzhelevski, M. Yuzhelevski and G. Jung, "Random telegraph noise analysis in time domain", Rev. of Sci. Instrum., vol. 71, no. 4, April 2000. DOI : $10.1063 / 1.1150519$

[24] V. Goiffon, G.R. Hopkinson, P.Magnan, F. Bernard, G. Rolland, O. SaintPe, "Multilevel RTS in Proton Irradiated CMOS Image Sensors Manufactured in a Deep Submicron Technology", IEEE Trans. Nucl. Sci., Vol.56, no. 4, Aug. 2009. DOI : 10.1109/TNS.2009.2014759.

[25] M. Guénin, S. Derelle, M. Caes, L. Rubaldo, I. Ribet-Mohamed, "RTS noise detection and voltage effect on RTS in HgCdTe focal plane array", J. Electron. Mater., June 2020. DOI : 10.1007/s11664-020-08271-y.

[26] R. Killick , P. Fearnhead, I. A. Eckley, "Optimal detection of changepoints with a linear computational cost", J. Am. Stat. Assoc., vol.107, no. 500, pp. 1590-1598, Dec. 2012. DOI : 10.1080/01621459.2012.737745.
Vignesh Arounassalame, received the Master degree in physics from the Institut d'Optique Graduate School, France, in 2018. He is currently pursuing the Ph.D. degree in optical engineering at ONERA, Palaiseau, France. His current research interests focus on electro-optical characterization of Type-II Superlattice photodetectors.

Maxence Guénin, a Grenoble INP Phelma graduate with a Nanoscience Physics Master diploma, is a Ph.D student at ONERA, Palaiseau, France. His studies mainly focus on the physical causes of the RTS phenomenon in $\mathrm{HgCdTe}$ cooler infrared sensors

Marcel Caes, received his $\mathrm{PhD}$ degree in 1992, for the electro-optical characterisation of infrared focal plane arrays under low photon flux, for astronomy applications. He was then hired at ONERA to conduct research in the field of infrared detectors. He is now one of the mainstays of the "Infrared Detectors Characterisation Lab" at ONERA, and focuses on the development of tools to analyse the experimental data.

Linda Höglund, Dr. Linda Höglund received a M.Sc. in Physics and Electrical Engineering 2001 and a Ph.D. in Material Science 2008 from Linköping University, Sweden. In 2001-2009, she worked at the Swedish research institute Acreo (now RISE), developing bolometers based on quantum structures and quantum dot infrared detectors. In 2009 she joined the Photonics group at NASA's Jet Propulsion Laboratory in Pasadena, California, USA. At JPL she was leading the optical characterization of type-II superlattice detector material. Since 2015 she has been working at IRnova, Sweden. At IRnova she is currently the R\&D Team manager, leading the development of novel T2SL and QWIP detectors.

Eric Costard, graduated as Doctor in Optics from the French University PARIS XI, joined Thales in 1985, where he has developed his background in semiconductor physics and processing. From the beginning of the $21^{\text {st }}$ century, he developed with his team innovation in infrared detection field: QWIP technology and InGaAs technology. With his team he has set up a pilot production line for QWIP FPAs for SWIR FPAs based on InGaAs material. Eric headed the IR Imaging Laboratory at III-VLab, he authored 15 patents in the field of QWIPs and received the Lamb Prize from the French Academy of Science in 2006. Subsequent to his work at Thales he also headed the III-V industrial transfer initiative at Sofradir in 2013. After many years working within the French IR industry, Eric moved to Sweden in the summer 2015 for joining IRnova. Eric is currently the CTO of IRnova where he continues to develop III-V detectors like QWIP and T2SL for gas detection and high end applications

Philippe Christol, is a professor of Electronic and Electrical Engineering at the Electronic Institute (IES) of Montpellier University, France, since 2005. He is specialist of infrared photodetection, in particular of antimonide-based photodetectors grown by Molecular Beam Epitaxy (MBE) on GaSb substrate. $\mathrm{He}$ is author/co-author of over 80 publications in refereed journals, a book chapter and contributed to over one hundred communications in international conferences including 15 invited talks. He has co-supervised $16 \mathrm{PhD}$ students in the past ten years. His research interests currently focus on electrical and optical properties of Ga-free InAs/InAsSb and Ga-containing InAs/GaSb XBn and XBp type-II superlattice barrier photodetectors operating from MWIR to VLWIR spectral domain

Isabelle Ribet-Mohamed graduated in 1998 as an optical engineer of the Institut d'Optique (IOGS) in France. She received her PhD degree from Paris Sud university in 2001, for the development of non linear optical diagnostic techniques based on optical parametric oscillators. She was then hired at ONERA to conduct research in the field of infrared detection and electro-optical systems. She is now in charge of the orientation of emerging infrared technologies. She is author/co-author of over 40 publications in refereed journals, a book chapter and contributed to over 80 communications in international conferences. She has co-supervised $8 \mathrm{PhD}$ students in the past 15 years. 